effects on bumblebee colonies exposed to neonicotinoids seem to be driven by impaired pollen foraging ${ }^{7,8}$ (leading to limited nutrition for larvae), the authors found no evidence that sulfoxaflor exposure caused significant differences in foraging performance. Perhaps early-stage colony growth and subsequent reproductive output were affected by sulfoxaflor toxicity to developing larvae, or by some other indirect mechanism - either way, the timing of declines in colony growth rate suggests that chronic sublethal stress at an early stage resulted in substantially reduced colony reproduction ${ }^{15}$.

Correctly determining the effects of insecticides relies on accurate assessments of exposure, which varies depending on whether chemicals are applied by spray, soil drench or seed treatment (Fig. 1). For example, spray applications can lead to relatively high levels of exposure for a few days, whereas seed treatments can result in low-level, chronic exposure through residues in nectar and pollen ${ }^{4,5}$. The authors based exposure to sulfoxaflor in their experiment on a scenario in which bees ingest nectar from crop flowers following a spray application - currently, the most common mode of application for this insecticide class.

However, this scenario discounts any exposure from contact with plant tissues or dietary exposure from crop pollen, and assumes that bees forage only on sulfoxaflortreated crops - all factors that could affect exposure levels. Moreover, exposure profiles would probably differ if sulfoxaflor were applied as a soil drench or seed treatment (an increasingly likely outcome following recent and probable future neonicotinoid regulation). Exposure could also be affected if sulfoxaflor, applied as a seed treatment or soil drench, moves outside crop fields and is absorbed by wild plants and contaminates their nectar and pollen, as reported for neonicotinoid seed treatments ${ }^{17,18}$. More data on sulfoxaflor concentrations in the nectar and pollen of bee-attractive crops are needed for an accurate assessment of the implications of sulfoxaflor use.

Nonetheless, Siviter et al. provide a valuable first step towards understanding the effects of sulfoxaflor exposure on bees. Future discussions must be broader than two-way comparisons of neonicotinoids and sulfoximines, because other classes of systemic insecticide (such as butenolides and anthranilic diamides) are also in agricultural use. It is vital to ascertain which of these insecticide classes represents the lowest potential risk to pollinators. A major part of the answer depends on how comparative risk assessments are undertaken, including which of the 20,000 living bee species are considered, because there is substantial variation in physiology, behaviour and ecology between these species. Such differences - particularly the extent to which species are social - might affect the bees' sensitivity to insecticides ${ }^{10,12,19}$. For instance, lowlevel insecticide exposure might have more impact on solitary bees than on highly social colonies that have an abundance of workers.

Finally, commercially reared pollinators (particularly honeybees) feature prominently in global agriculture, but cannot provide all of the crop-pollination services needed ${ }^{20}$. Wild pollinators, including bumblebees and solitary bees, have a crucial, undervalued role that is likely to become increasingly important as our crop-pollination demands rise ${ }^{1,20}$. Our understanding of the risks to pollinators, and the choices we make about pest control, must evolve to reflect and balance these realities. There are no risk-free choices, but with more information such as that provided by Siviter and colleagues, we can make the most appropriate decisions about how to produce the food we need without inflicting irreparable damage on the global environment and the essential ecosystem services (such as pollination) on which we depend.

Nigel E. Raine is at the School of Environmental Sciences, University of Guelph, Guelph, Ontario N1G 2W1, Canada. e-mail:nraine@uoguelph.ca

1. Garibaldi, L. A. et al. Science 339, 1608-1611 (2013).

2. Vanbergen, A. J. \& the Insect Pollinators Initiative. Front. Ecol. Environ. 11, 251-259 (2013).
3. Siviter, H., Brown, M. J. F. \& Leadbeater, E. Nature 561, 109-112 (2018)

4. Godfray, H. C. J. et al. Proc. R. Soc. B 281, 20140558 (2014).

5. Godfray, H. C. J. et al. Proc. R. Soc. B 282, 20151821 (2015).

6. Stanley, D. A. \& Raine, N. E. Funct. Ecol. 30, 1132-1139 (2016).

7. Gill, R. J., Ramos-Rodriguez, O.\& Raine, N. E. Nature 491, 105-108 (2012).

8. Stanley, D. A., Russell, A. L., Morrison, S. J., Rogers, C. \& Raine, N. E. J. Appl. Ecol. 53, 1440-1449 (2016).

9. Whitehorn, P. R., O'Connor, S., Wackers, F. L. \& Goulson, D. Science 336, 351-352 (2012).

10.Rundlöf, M. et al. Nature 521, 77-80 (2015).

11.Baron, G. L., Jansen, V. A. A., Brown, M. J. F. \& Raine, N. E. Nature Ecol. Evol. 1, 1308-1316 (2017).

12.Woodcock, B. A. et al. Science 356, 1393-1395 (2017).

13.Stanley, D. A. et al. Nature 528, 548-550 (2015).

14.Brown, M. J. F. et al. PeerJ 4, e2249 (2016).

15.Bryden, J., Gill, R. J., Mitton, R. A. A., Raine, N. E. \& Jansen, V. A. A. Ecol. Lett. 16, 1463-1469 (2013).

16.Ellis, C., Park, K. J., Whitehorn, P., David, A. \& Goulson, D. Environ. Sci. Technol. 51, 1727-1732 (2017).

17. Tsvetkov, N. et al. Science 356, 1395-1397 (2017).

18. Nicholls, E. et al. Environ. Sci. Technol. https://doi. org/10.1021/acs.est.7b06573 (2018).

19.Arena, M. \& Sgolastra, F. Ecotoxicology 23, 324-334 (2014).

20.Aizen, M. A. \& Harder L. D. Curr. Biol. 19, 915-918 (2009).

This article was published online on 15 August 2018.

\title{
Spotlight on proteins that aid malaria
}

The multiprotein complex PTEX enables malaria-causing parasites to survive inside red blood cells. Studies reveal how PTEX assembles, and identify a function for one of the complex's proteins, EXP2. SEE ARTICLE P.70

\section{TANIA F. DE KONING-WARD}

$\mathrm{M}$ alaria is caused by the parasite Plasmodium falciparum. For part of its life cycle, this organism resides inside human red blood cells in a membranebound compartment called a vacuole. To survive, multiply and evade an immune response in this environment, $P$. falciparum must transport nutrients and proteins across the vacuolar membrane ${ }^{1}$. On page 70 , Ho et al. ${ }^{2}$ report the structure of the parasite PTEX complex, which resides on the vacuolar membrane and facilitates the export of proteins from the vacuole to the cytoplasm of red blood cells ${ }^{3}$. And in a paper in Nature Microbiology, Garten et al. ${ }^{4}$ reveal that the protein EXP2, which forms part of the PTEX protein-conducting channel located in the vacuolar membrane, can also form a channel that facilitates nutrient transfer across the membrane. These insights into the structure and function of key proteins that aid the survival of $P$. falciparum might help efforts to develop new antimalarial drugs.

PTEX consists of five proteins ${ }^{3}$ : HSP101, PTEX150, EXP2, PTEX88 and TRX2. Multiple HSP101, PTEX150 and EXP2 molecules assemble to form the core part of PTEX ${ }^{3,5}$. It has been predicted that HSP101 unfolds proteins destined for export, and provides the energy needed for cargo to pass through the vacuolarmembrane-spanning part of the channel, which is proposed ${ }^{3,6}$ to consist of EXP2. PTEX150 is thought $^{5}$ to have a structural role, connecting HSP101 and EXP2.

Reduced expression ${ }^{7}$ of HSP 101 or PTEX150, or inhibition ${ }^{8}$ of the assembly of HSP101 into the PTEX complex, results in parasite death. PTEX is specific to species of the genus Plasmodium and is not made by humans. It is an attractive drug target because it provides the only known route by which parasite proteins enter the cytoplasm of a red blood cell. However, PTEX's relative 


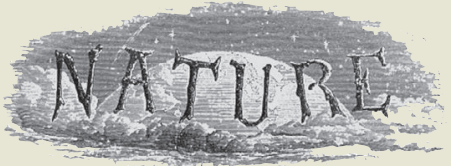

50 Years Ago

Everybody knows, of course, that there is a genuine and unavoidable conflict between the functions of museums as centres of scholarship and as places of entertainment. Curators can be forgiven for wishing that visitors would let them get on with serious work. In practice, the Science Museum seems to have mastered these yearnings quite successfully, and in the past few months there have been some welcome signs of an anxiety to please ... Yet there is a long way to go before the museum shoulders wholeheartedly its responsibility for seeing that people, and particularly young people, are provided with a vivid and contemporary vision of what science is like. Even the new children's exhibition will not let the little creatures know about electronic computers, for example.

From Nature 7 September 1968

\section{Years Ago}

Considerable interest was taken last week in the demonstrations of "reading by ear" at the British Scientific Products Exhibition. The original construction of Dr. Fournier d'Albe's “type-reading optophone" ... has recently been modified by replacing the Nernst lamp by a small drawn-wire lamp, and by arranging the whole apparatus in such a manner that any ordinary book or newspaper can be inserted and read without cutting it up into pages or columns. The demonstrations consisted in taking an ordinary book ..., opening it at random ... and asking the blind pupil to read a few words or lines ... By a curious coincidence the first words thus read were "in the light". The reader, a girl of nineteen blind from early infancy, was the first blind person to read by ear.

From Nature 5 September 1918

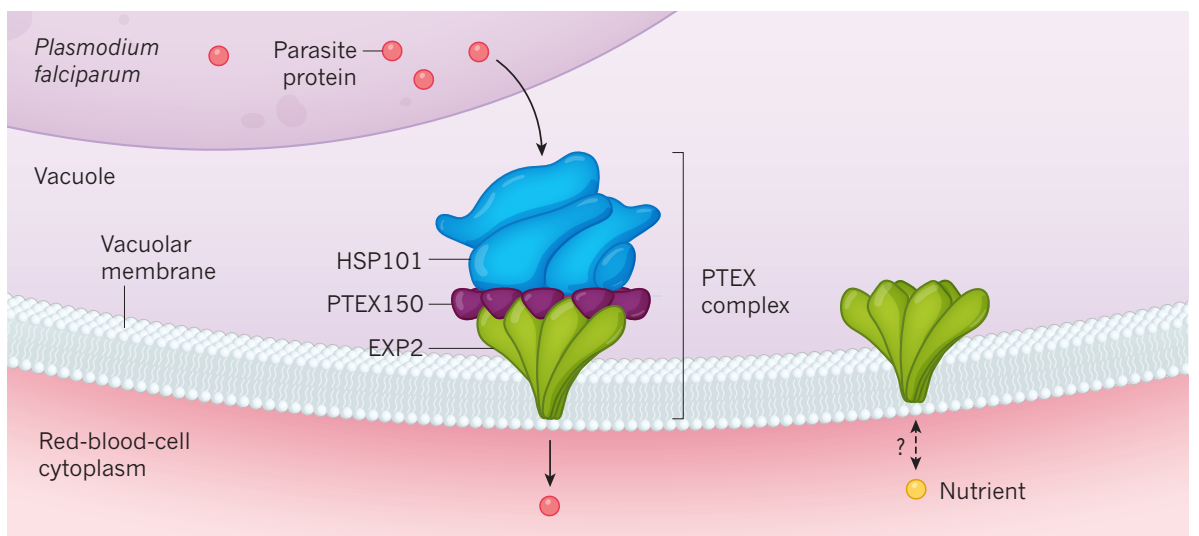

Figure 1 | Proteins that are essential for the malaria parasite's survival. During part of its life cycle, the Plasmodium falciparum parasite resides inside a membrane-bound vacuole in human red blood cells. Ho et al. ${ }^{2}$ report the structure of a parasitic multiprotein complex called PTEX, which is present on the inside of the vacuolar membrane. This complex is essential for parasite survival, and proteins are exported from the vacuole through PTEX into the cytoplasm of red blood cells. The authors' analysis provides a detailed view of three of the proteins that form PTEX: EXP2, HSP101 and PTEX150 (only 20\% of the structure of PTEX150 was determined). The authors' work also illuminates how proteins transit through the PTEX complex. Garten et al. ${ }^{4}$ report that EXP2 can form a channel that enables nutrients to be transported across the vacuolar membrane. Whether this occurs in both directions, or in only one, is not known.

novelty offers few clues to how it functions. EXP2 synthesized in the laboratory can form protein channels in lipid bilayers ${ }^{9}$. However, there have been no reports of fulllength HSP101 or PTEX150 having been successfully synthesized for use in in vitro experiments. This has prevented structural analysis of the proteins, or reconstitution of the core PTEX complex in lipid membranes, to determine how the complex assembles and functions.

Because of these experimental limitations, Ho et al. ${ }^{2}$ opted instead to extract PTEX directly from red blood cells containing the parasite. Then, using a technique called cryo-electron microscopy (cryo-EM), the authors captured two distinct structural conformations of the core PTEX complex in the process of exporting unfolded protein cargo - they called these conformations the 'engaged' and 'resetting' states. The cryo-EM analysis revealed that HSP101, PTEX150 and EXP2 assemble into an asymmetrical structure containing six molecules of HSP101, seven of PTEX150 and seven of EXP2. These structures closely align with models of the organization and size of PTEX that had been predicted from biochemical and proteinanalysis experiments ${ }^{3,5}$.

Ho and colleagues found that the seven EXP2 molecules, which make up the protein channel in the lipid membrane, create a funnel shape, with the amino terminus of each molecule forming a transmembrane helix in the vacuolar membrane to provide an anchoring 'stem' (Fig. 1). The 'mouth' of EXP2 constitutes the bulk of the protein, and faces into the vacuole. This end of EXP2 contains a domain that tethers it to the carboxy-terminal domain of HSP101, situated directly on top. Only approximately $20 \%$ of the structure of PTEX150 could be determined. Nevertheless, this was sufficient to reveal that each PTEX150 molecule slots in between adjacent EXP2 molecules at the mouth of the EXP2 funnel, curling down towards the stem. Thus, PTEX150 provides a protective path for unfolded protein cargo transiting from HSP101 to EXP2.

Of the three proteins, HSP101 displayed the greatest structural difference between the engaged and resetting states of PTEX, and on this basis the authors propose a mechanism for how cargo is threaded through PTEX's central cavity. In this model, domains of the six assembled HSP101 molecules form two 'hands' that work together to thread unfolded cargo through the PTEX150 and EXP2 funnel. In the engaged state of PTEX, both the 'active' and 'passive' hands of HSP101 grasp the unfolded cargo. The cargo is then fed downwards through the central cavity of PTEX in a spiral fashion as it passes from the active to the passive hand. In the resetting state, HSP101's active hand moves upwards to grasp the next section of the cargo protein for transport, and the passive hand grips the cargo to prevent it from slipping backwards and away from the PTEX channel.

The cryo-EM structures provide insight into several crucial interactions between the PTEX components. These interactions are potentially required for assembly and optimal function of the complex, and could be tested using genetic approaches to validate the model. Ho and colleagues were unable to determine the structure of the N-terminal domain of HSP101 that binds the protein cargo. Thus, it is unclear how cargo is recognized by HSP101, and whether cargo proteins are unfolded by proteins known as chaperones before they reach PTEX. Given that unfolded proteins pass through PTEX, these cargo proteins would then need to be refolded to function, presumably by other chaperone proteins. However, because EXP2 does not 
extend into the cytoplasm of red blood cells, it is unclear how chaperone proteins in the host cell might be recruited to cargo exiting PTEX.

Garten et al. ${ }^{4}$ investigated EXP2 using in vitro experiments, and report that it has another role in addition to its function in PTEX. Previous experiments using electrophysiological techniques have shown that a channel exists in the vacuolar membrane of parasite-infected red blood cells through which nutrients such as amino acids and sugars can pass ${ }^{10}$, but the identity of this channel has been a mystery. In electrophysiological studies, Garten and colleagues demonstrated a direct relationship between the level of expression of EXP2 and the frequency of detection of the mysterious channel. When the authors generated a version of EXP2 that had a truncated C-terminal domain, which is located in the vacuole and is not required for protein export, this altered the voltage-response properties of the nutrient channel, leading the authors to conclude that EXP2 is indeed the elusive nutrient channel.

That EXP2 might have a role separate from its function in PTEX is consistent with evidence that EXP2's gene-expression profile differs from that of the other PTEX components ${ }^{5}$. Moreover, the authors found that most EXP2 is not present in a complex with PTEX. Although EXP2 is essential for parasite survival ${ }^{11,12}$, the contribution of the EXP2 nutrient channel to parasite growth remains unknown. The channel could be characterized in detail if EXP2 was incorporated into lipid bilayers for in vitro experiments.

The studies by Ho, Garten and their respective colleagues offer a close look at how major $P$. falciparum proteins function. Interestingly, EXP2 is evolutionarily conserved among vacuolar-dwelling parasites called apicomplexans ${ }^{1}$. Perhaps the nutrient-transiting capacity of EXP2 was adapted by $P$. falciparum to generate a protein-conducting channel that evolved through the recruitment of other proteins such as HSP101 and PTEX150. EXP2 and PTEX are expressed throughout the life cycle of $P$. falciparum, so drugs that target them might be highly effective at tackling malaria. These new insights into the interactions between the components of PTEX offer exciting possibilities for the development of peptides or small molecules that might block the function of this complex.

Tania F. de Koning-Ward is in the School of Medicine, Deakin University, Geelong, Victoria 3216, Australia.

e-mail:tania.dekoning-ward@deakin.edu.au

1. de Koning-Ward, T. F. et al. Nature Rev. Microbiol. 14 494-507 (2016).

2. Ho, C.-M. et al. Nature $561,70-75$ (2018).

3. de Koning-Ward, T. F. et al. Nature 459, 945-949 (2009).

4. Garten, M. et al. Nature Microbiol. http://doi. org/10.1038/s41564-018-0222-7 (2018).

5. Bullen, H. E. et al. J. Biol. Chem. 287, 7871-7884 (2012).

6. Johnson, D. et al. Parasitology 109, 1-9 (1994).

7. Elsworth, B. et al. Nature 511, 587-591 (2014)

8. Beck, J. R., Muralidharan, V., Oksman, A. \& Goldberg, D. E. Nature 511, 592-595 (2014).

9. Hakamada, K., Watanabe, H., Kawano, R., Noguchi, K. \& Yohda, M. Biochem. Biophys. Res. Commun. 482, 700-705 (2017).

10.Desai, S. A., Krogstad, D. J. \& McCleskey, E. W. Nature 362, 643-646 (1993).

11. Matthews, K. et al. Mol. Microbiol. 89, 1167-1186 (2013).

12.Matz, J. M., Matuschewski, K. \& Kooij, T. W. A. Mol. Biochem. Parasitol. 191, 44-52 (2013).

This article was published online on 27 August 2018.

\section{Designer atom arrays for quantum computing}

A key step in the development of quantum computers that use neutral atoms as quantum bits is the assembly of tailored 3D arrays of atoms. Two laser-based approaches have now been reported to do this. SEE LETTERS P.79 \& P.83

\section{NATHAN LUNDBLAD}

$\mathrm{Q}$ uantum computers and simulators are of enormous interest because of their potential to shed light on mysteries of physics that are difficult to model using conventional computers. Some physical platforms used in realizing quantum-computing protocols - including trapped ions and several solid-state systems based on superconductors - have received increased attention in the past year. But in this issue, two groups report technical breakthroughs that will aid the development of another platform: trapped neutral atoms. Barredo et al. ${ }^{1}$ (page 79) report their use of precision optical-engineering methods to sort atoms into arbitrary $3 \mathrm{D}$ patterns, whereas Kumar et al. ${ }^{2}$ (page 83) construct cubic lattices by revisiting a fanciful thought experiment known as Maxwell's demon. The ability to organize neutral atoms exactly into planned 3D arrays will be valuable for the development of neutral-atom quantum computers that use a large number of quantum bits (qubits).

Arrays of isolated neutral atoms have long shown promise for quantum computing because neutral-atom qubits are extremely well isolated from environmental noise and are highly controllable, and also because such systems can be scaled up to large numbers of qubits $^{3,4}$. Given that controlled interactions between atoms are needed to perform quantum-computing operations, neutral-atom quantum computers will need qubits to be precisely arranged in a specified pattern. However,
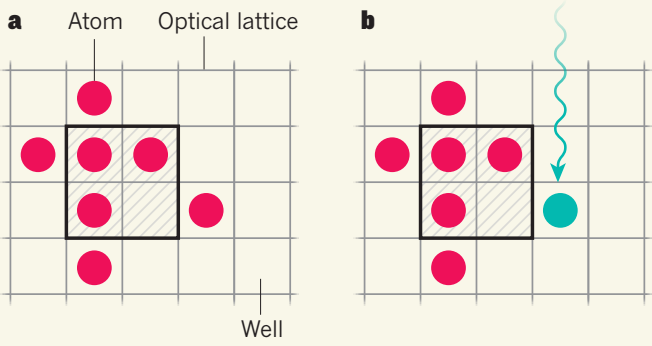

Figure 1 A protocol for arranging neutral atoms in cubic optical lattices. Kumar et al. ${ }^{2}$ report a method for arranging ultracold, neutral caesium atoms in defined patterns in a cubic, 3D optical lattice - a series of laser-generated potential-energy wells in which atoms can be confined. Only one layer of atoms is shown, for simplicity. a, The atoms start off in random positions and in the same electronic state (state A, red). The shaded square indicates a target region c

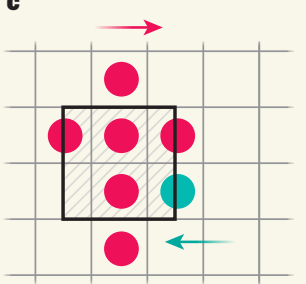

d

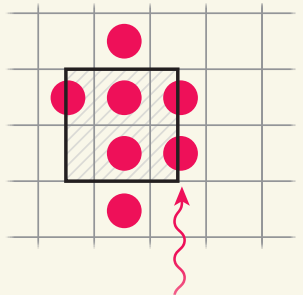

e

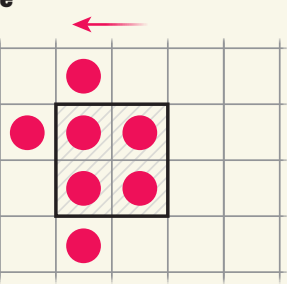

that is to be filled with atoms. $\mathbf{b}, \mathrm{A}$ combination of lasers and microwaves (wavy arrow) flips the state of one atom into a different state (state $B$, turquoise) c, A lattice shift is induced that moves the lattice and all atoms in state $\mathrm{A}$ half a step to the right and those in state B half a step to the left. $\mathbf{d}$, The atom in state B is flipped back to state A. e, A reverse lattice shift moves the lattice and all atoms in state $\mathrm{A}$ half a step to the left, so that the square region is now filled with atoms. 